TRANSACTIONS OF THE

AMERICAN MATHEMATICAL SOCIETY

Volume 355, Number 4 , Pages 1559-1577

S 0002-9947(02)03168-9

Article electronically published on November 18, 2002

\title{
LOCAL GEOMETRY \\ OF SINGULAR REAL ANALYTIC SURFACES
}

\author{
DANIEL GRIESER
}

\begin{abstract}
Let $V \subset \mathbb{R}^{N}$ be a compact real analytic surface with isolated singularities, and assume its smooth part $V_{0}$ is equipped with a Riemannian metric that is induced from some analytic Riemannian metric on $\mathbb{R}^{N}$. We prove:

(1) Each point of $V$ has a neighborhood which is quasi-isometric (naturally and "almost isometrically") to a union of metric cones and horns, glued at their tips.

(2) A full asymptotic expansion, for any $p \in V$, of the length of $V \cap\{q$ : $\operatorname{dist}(q, p)=r\}$ as $r \rightarrow 0$.

(3) A Gauss-Bonnet Theorem, saying that each singular point contributes $1-l /(2 \pi)$, where $l$ is the coefficient of the linear term in the expansion of $(2)$.

(4) The $L^{2}$ Stokes Theorem, selfadjointness and discreteness of the LaplaceBeltrami operator on $V_{0}$, an estimate on the heat kernel, and a GaussBonnet Theorem for the $L^{2}$ Euler characteristic.

As a central tool we use resolution of singularities.
\end{abstract}

\section{INTRODUCTION}

In this paper we investigate some aspects of the geometry and analysis of a compact real analytic surface with isolated singularities. By this we mean a compact subset $V$ of some $\mathbb{R}^{N}$ which is locally given as the zero set of a finite number of real analytic functions, and such that $V$ is a smooth 2-dimensional manifold outside a finite set, $\operatorname{sing} V$. Let $V_{0}=V \backslash \operatorname{sing} V$ be the smooth part. We assume that $V$ has no isolated points, i.e., $\overline{V_{0}}=V$. In order to speak of geometry or analysis on $V$, we need to introduce a metric. We assume that an induced metric $g$ is given on $V$, i.e., a Riemannian metric on $V_{0}$ which arises as the restriction of some real analytic Riemannian metric on $\mathbb{R}^{N}$. The metric $g$ induces an (intrinsic) distance function $d$ on $V$.

Our first concern is a description of the local structure of the metric space $(V, d)$ up to quasi-isometry. Here, two metric spaces $(X, d),\left(X^{\prime}, d^{\prime}\right)$ are called quasiisometric if there is a bijective map $\Phi: X \rightarrow X^{\prime}$ that is a quasi-isometry, i.e., such

Received by the editors July 9, 2002.

2000 Mathematics Subject Classification. Primary 14P15; Secondary 32B20, 53B20, 58J99.

Key words and phrases. Real analytic sets, quasi-isometry, Gauss-Bonnet theorem, $L^{2}$ Stokes theorem, resolution of singularities.

The author gratefully acknowledges support by the Deutsche Forschungsgemeinschaft (Gerhard-Hess-Programm) and the Erwin Schrödinger Institute.

(C)2002 American Mathematical Society 
that there is a constant $C>0$ with

$$
C^{-1} d(x, y) \leq d(\Phi(x), \Phi(y)) \leq C d(x, y) \quad \text { for all } x, y \in X .
$$

The model metric spaces that will occur are cones and horns. These are defined as follows: Let $\gamma \geq 1$ and $\varepsilon>0$. The space

$$
C_{\gamma}=\left([0, \varepsilon)_{r} \times S_{\theta}^{1}\right) /\left(\{0\} \times S^{1}\right)
$$

with distance function $d_{\gamma}$ induced by the Riemannian metric

$$
d r^{2}+r^{2 \gamma} d \theta^{2}
$$

for $r>0$ and $d_{\gamma}(0,(r, \theta))=r$, where $0:=\left[\{0\} \times S^{1}\right]$ is the "tip", is called a $\gamma$-horn, and $\gamma$ is called its exponent. A 1-horn is also called a cone.

Theorem 1.1. Let $(V, d)$ be as above. Then each $p \in V$ has a neighborhood (in $V$ ) that is quasi-isometric to a union of finitely many cones and horns with rational exponents, glued at their tips.

The distance function $d$ on the space glued from $C_{\gamma_{1}}, \ldots, C_{\gamma_{n}}$ is given by the $d_{\gamma_{i}}$ on each $C_{\gamma_{i}}$ and $d(p, q)=d_{\gamma_{i}}(p, 0)+d_{\gamma_{j}}(0, q)$ if $p \in C_{\gamma_{i}}, q \in C_{\gamma_{j}}, i \neq j$.

Example. If $a \leq b$ are positive integers, then the set

$$
\left\{(x, y, z):\left(x^{2}+y^{2}\right)^{a}=z^{2 b}\right\} \subset \mathbb{R}^{3}
$$

with metric induced from the Euclidean metric on $\mathbb{R}^{3}$ is quasi-isometric (near zero) to two $(b / a)$-horns glued at their tips. From this example one sees that any combination of cones and horns can occur if one allows $V$ to be semi-analytic (i.e., allowing inequalities in a local description of $V$ ) or even semi-algebraic. So Theorem 1.1 gives a complete classification in this case.

Note that a small ball around a smooth point is quasi-isometric to a Euclidean ball, which is a cone in polar coordinates.

Theorem 1.1 can be sharpened considerably: First, the quasi-isometry can be chosen to be "natural", and second, it gets closer and closer to an isometry as one approaches $p$. Before we state this precisely, we introduce the infinitesimal notion of quasi-isometry: Two Riemannian metrics $g, g^{\prime}$ on a manifold $M$ are called quasiisometric if there is $C>0$ such that for each $x \in M$ one has the inequality of quadratic forms

$$
C^{-2} g_{x} \leq g_{x}^{\prime} \leq C^{2} g_{x}
$$

If this holds, then we write

$$
g \approx_{C} g^{\prime}
$$

or simply $g \approx g^{\prime}$. $C$ is called the quasi-isometry constant. It is easy to see that, for two connected Riemannian manifolds $(M, g),(N, h)$, a diffeomorphism $\Phi: M \rightarrow N$ is a quasi-isometry for the induced distance functions if and only if $\Phi^{*} h \approx g$.

Let dist denote the (extrinsic) distance function on $\mathbb{R}^{N}$, and define $B_{\varepsilon}(p)=\{x \in$ $V: \operatorname{dist}(x, p)<\varepsilon\}$.

Theorem 1.2. Let $(V, g)$ be as above and let $p \in V$. If $\varepsilon_{0}$ is sufficiently small, then the following holds for each connected component $K$ of $B_{\varepsilon_{0}}(p) \backslash p$ :

There is a parametrization

$$
\Phi:\left(0, \varepsilon_{0}\right) \times S^{1} \rightarrow K
$$


that "preserves" r, i.e.,

$$
\operatorname{dist}(\Phi(r, \theta), p)=r \quad \text { for all } r<\varepsilon_{0},
$$

and is normalized arc length along each $K_{r}:=\{q \in K$ : dist $(p, q)=r\}$ such that

$$
\Phi^{*} g \approx_{1+\delta} d r^{2}+\left(\frac{l(r)}{2 \pi}\right)^{2} d \theta^{2} .
$$

Here, $l(r)$ is the length of $K_{r}$. In (2), one can choose

$$
\delta=C \varepsilon^{\alpha}
$$

when restricting $\Phi$ to $(0, \varepsilon) \times S^{1}, \varepsilon \leq \varepsilon_{0}$, with $C$ and $\alpha>0$ only depending on $V$ and $g$.

Explicitly, the arc length condition on $\Phi$ means

$$
|\partial \Phi / \partial \theta|_{g}=l(r) /(2 \pi) .
$$

Clearly, a parametrization $\Phi$ satisfying (1) and (4) (if it exists) is uniquely determined by one curve $r \mapsto \Phi\left(r, \theta_{0}\right)$ and an orientation on $K$.

In order to deduce Theorem 1.1 from Theorem 1.2 one needs to know that $l(r)$ is of order $r^{\gamma}$ for a rational $\gamma \geq 1$, as $r \rightarrow 0$. The following theorem gives a much more precise result.

Theorem 1.3. The function $l(r)$ defined in Theorem 1.2 has an asymptotic expansion, as $r \rightarrow 0$,

$$
l(r) \sim \sum_{i, j} C_{i, j} r^{i}(\log r)^{j}
$$

with $i$ ranging over $n / m \geq 1$ for some fixed integer $m$ and arbitrary integers $n \geq m$, and $j$ ranging over $\{0,1\}$. Also, the leading term contains no logarithm, i.e., if

$$
\gamma=\min \left\{i: C_{i, 0} \neq 0 \text { or } C_{i, 1} \neq 0\right\},
$$

then $C_{\gamma, 1}=0$.

The asymptotic expansion (5) may be differentiated (arbitrarily often) term by term.

We use these precise descriptions of the local geometry to draw some conclusions of a global nature. First, we generalize the classical Gauss-Bonnet theorem.

Theorem 1.4 (Gauss-Bonnet Theorem). Let $V$ be a compact real analytic surface with isolated singularities $p_{1}, \ldots, p_{R}$ with an induced Riemannian metric on $V_{0}=$ $V \backslash\left\{p_{1}, \ldots, p_{R}\right\}$ as above. Let $\mathcal{K}$ denote the Gauss curvature. According to Theorem 1.1, sufficiently small pointed neighborhoods of the $p_{i}, i=1, \ldots, R$, have connected components that are quasi-isometric to cones or horns. Let $l_{1}, \ldots, l_{S}$ be the leading coefficients in the length expansions (Theorem 1.3) for all the cone-like components.

Then $\mathcal{K}$ is integrable over $V_{0}$, and

$$
\chi(V)=R+\frac{1}{2 \pi} \int_{V_{0}} \mathcal{K}-\frac{1}{2 \pi} \sum_{i=1}^{S} l_{i},
$$

where $\chi(V)$ is the Euler characteristic. 
We now address some questions of analysis. The general problem here is the following: Given a Riemannian manifold $V_{0}$ which is the smooth part of a compact "singular space" $V$, e.g. an analytic variety, how do the properties of the natural differential operators defined on $V_{0}$ differ from those in the smooth case (i.e., $V=$ $\left.V_{0}\right)$ ? Here the "properties" can be of a very basic nature, like (i) below, or rather involved, like (iii). The Riemannian metric $g$ induces a scalar product on differential forms. By $L^{2}\left(\bigwedge V_{0}\right)$ we denote the completion of the space of compactly supported smooth forms on $V_{0}$ under this norm. The exterior derivative $d$ and its transpose $d^{t}$ act on elements of $L^{2}\left(\bigwedge V_{0}\right)$ in the sense of distributions.

Theorem 1.5. Let $(V, g)$ be as in Theorem 1.4.

(i) The $L^{2}$ Stokes theorem holds, i.e., if $\omega, \eta, d \omega, d^{t} \eta \in L^{2}\left(\bigwedge V_{0}\right)$, then

$$
(d \omega, \eta)=\left(\omega, d^{t} \eta\right) .
$$

(ii) The Gauss-Bonnet operator $D_{G B}=d+d^{t}$ and the Laplace-Beltrami operator $\Delta=D_{G B}^{2}$, defined on $C_{0}^{\infty}\left(\bigwedge V_{0}\right)$, have natural selfadjoint extensions as unbounded operators in $L^{2}\left(\bigwedge V_{0}\right)$. Their spectra are discrete.

(iii) The heat kernel $e^{-t \Delta}$ of the Laplace-Beltrami operator in (ii) is trace class, and

$$
\operatorname{tr} e^{-t \Delta} \leq \frac{C}{t}
$$

(iv) The $L^{2}$ Euler characteristic of $V$, i.e., the index of the operator $D_{G B}$ acting from even forms in $\mathcal{D}\left(D_{G B}\right)$ to odd forms, equals

$$
\chi_{(2)}(V)=N+\frac{1}{2 \pi} \int_{V_{0}} \mathcal{K}-\frac{1}{2 \pi} \sum_{i=1}^{S} l_{i},
$$

where $N$ is the total number of cones and horns that occur near singularities of $V$.

(i) is equivalent to a property called "uniqueness of ideal boundary conditions", see [20. It says that no boundary terms occur in the integration by parts that is implicit in (6). It is conjectured to be true for all complex projective varieties with induced metrics; see [16] for related material. However, it is not true in general for compact real algebraic varieties, even if the singularities are isolated, see [20].

Note that (ii) does not say that $\Delta$ is essentially selfadjoint on $C_{0}^{\infty}\left(\bigwedge V_{0}\right)$. In fact, it need not be, as one can see for certain cones.

We expect that (iii) can be sharpened considerably by a more refined analysis. For this analysis we expect the "almost isometry" estimate (3) to be crucial.

Outline of the proofs. The asserted quasi-isometry in Theorem 1.2 with any constant (resp. with the constant $1+O\left(\varepsilon^{\alpha}\right)$ ) holds iff both of the following two statements are true (this is elementary; for a proof see Lemmas 3.1 and 3.2):

(1) The angle $\beta$ between the curves $\theta=$ const and the curves $r=$ const on $K$ is bounded away from zero as $r \rightarrow 0$ (resp. tends to a right angle, with an error of order $r^{\alpha}$ ).

(2) The length $\left|\Phi_{r}\right|$ is bounded (resp. is $1+O\left(r^{\alpha}\right)$ ).

We denote $\Phi_{r}=\partial \Phi / \partial r$, etc. For simplicity, we assume in this outline that $p=0$ and $g$ is induced from the Euclidean metric. Now $\beta=\angle\left(\Phi_{r}, \Phi_{\theta}\right)$. If we write $\Phi(r, \theta)=r \phi(r, \theta)$ with $|\phi|=1$, then $\Phi_{r}=\phi+r \phi_{r}$ is a decomposition into a "radial" 
component $\phi$ that is orthogonal to $\Phi_{\theta}$ and a "spherical" component $r \phi_{r}$. Therefore, the quasi-isometry is equivalent to the estimate $r\left|\phi_{r}\right|<C$ (resp. $r\left|\phi_{r}\right|<C r^{\alpha}$ ).

Since the arc length parametrization is difficult to handle directly, we deal first with arbitrary parametrizations $\Psi=r \psi,|\psi|=1$, and show (in Section 3) that the $r\left|\phi_{r}\right|$-estimate follows from the analogous $r\left|\psi_{r}\right|$-estimate plus the uniform boundedness, as $r \rightarrow 0$, of the integrals over $K_{r}$ of the absolute geodesic curvature of $K_{r}$ in $K$. The curvature estimate reduces to a length estimate in the unit tangent bundle, which can be proved by standard analytic techniques, see Section 4

The problem is, then, to construct $\psi$ satisfying $r\left|\psi_{r}\right|<C r^{\alpha}$. This is easy in the model case of a family of curves given by hyperbolas, i.e., the family $x^{a} y^{b}=r$ in the $(x, y)$-plane, for positive integers $a$ and $b$. Using resolution of singularities, we can reduce the general case to this model. This will prove Theorem 1.2, see Section 2

The asymptotic analysis of $l(r)$ (Section 5 ) is also most easily done on the blownup space obtained by resolution of singularities. The curves $K_{r}$ are then, locally, simply hyperbolas as above, but their length is measured with respect to a degenerating semi-Riemannian metric. In such a general situation, one always has an asymptotic expansion as stated, although the leading term may be $r^{\gamma} \log r$. We prove this using the Mellin transform, which reduces the problem to analytic continuation in the complex variable $z$ of an integral of the form $\int_{\mathbb{R}^{2}}|q(x)|^{z+A}|p(x)|^{1 / 2} \chi(x) d x$ for real analytic functions $p, q$ and $\chi \in C_{0}^{\infty}\left(\mathbb{R}^{2}\right)$, plus a decay estimate for large $\operatorname{Im} z$. To do this, we adapt an idea by Bernstein and Gel'fand 2] and Atiyah [1, using resolution of singularities again. A separate consideration is needed to exclude a logarithm in the leading term, in our situation.

The Gauss-Bonnet Theorem is proved (in Section 6) by first excising small neighborhoods of the singular points and applying the classical Gauss-Bonnet Theorem to the resulting smooth manifold with boundary. The boundary integrals of geodesic curvature are interpreted as arc length variation. Here one needs the differentiated form of (the first term of) the asymptotics in Theorem 1.3 The integrability of $\mathcal{K}$ follows again by a standard argument, bounding $|\mathcal{K}|$ by the area element of the unit normal bundle of $V$.

Theorem 1.5 follows easily from quasi-isometry invariance of some of the assertions and well-known results in the conic and horn-like cases. This is done in Section 7

Further remarks and related work. All results are true for sub-analytic sets $V$ as well, since the methods extend to this case. Clearly, the assumption $V \subset \mathbb{R}^{N}$ was only made for notational convenience. All that matters is that the Riemannian metric on $V_{0}$ is locally induced by some local embedding. In any case, at least any smooth real analytic manifold can be embedded analytically in $\mathbb{R}^{N}$ (see [23]). So probably the assumption $V \subset \mathbb{R}^{N}$ is no loss of generality.

Some authors have obtained results similar to ours: L. Birbrair [5] classifies compact sub-analytic sets locally up to quasi-isometry, by methods very different from ours ("pancake decomposition"), for $g$ the Euclidean metric. However, these methods are not precise enough to yield our sharper Theorem[1.2. As observed by A. Parusiński, the expansion in Theorem 1.3 also follows from a general result by J.-M. Lion and J.-P. Rolin [26], again proved by different methods; however, the statement about the leading term cannot be obtained from [26]. The local geometry 
of semi-algebraic sets is also studied in [6]. For more on curvature of sub-analytic sets see J. Fu [18].

If $V$ is a complex algebraic curve, then it falls in the class of surfaces considered here. In this case, Theorem 1.1 is much easier to prove. Questions of analysis have been treated in great detail by Nagase [27, Brüning and Lesch [11, [10] and Brüning, Peyerimhoff, and Schröder [12.

The author wishes to thank M. Lesch for many helpful discussions and for suggesting work on the spectral theoretic problems that motivated this study, and P. Milman for clarifying some questions on the resolution of singularities. Also, thanks to J. Fu and J. Tolksdorf for interesting discussions on the subject.

\section{LOCAL METRIC STRUCTURE}

In this section we carry out the main steps in the proof of Theorem 1.2, deferring the proofs of some auxiliary results to later sections.

We will use geodesic normal coordinates around $p$ in $\mathbb{R}^{N}$; these are given by the smooth map

$$
P C:[0, \infty) \times S^{N-1} \rightarrow \mathbb{R}^{N}
$$

defined by $P C(r, \omega)=\gamma(r)$, where $\gamma$ is the geodesic with $\gamma(0)=p, \gamma^{\prime}(0)=\omega$. $S^{N-1}$ denotes the unit sphere in $T_{p} \mathbb{R}^{N}$ with respect to $g_{\mid p}$, where we denote by $g$ the metric on $\mathbb{R}^{N}$ also. For $r$ near zero, the form of the metric in normal coordinates is

$$
P C^{*} g=d r^{2}+r^{2} g_{S^{N-1}}(r),
$$

where $g_{S^{N-1}}(r)$ is a family of Riemannian metrics on $S^{N-1}$ that depends smoothly on $r \geq 0$. We will denote this metric on $S^{N-1}$ simply by $|\cdot|$.

We consider local parametrizations $\Psi$ of $K$ that satisfy (1), i.e., that are of the form

$$
\begin{aligned}
\Psi:(0, \varepsilon) \times(-A, A) & \rightarrow K, \\
(r, s) & \mapsto P C(r, \psi(r, s)),
\end{aligned}
$$

for some $A>0, \varepsilon \leq \varepsilon_{0}$ and some map $\psi:(0, \varepsilon) \times(-A, A) \rightarrow S^{N-1}$.

The following proposition reduces the desired quasi-isometry statement to an estimate on derivatives of such parametrizations $\Psi$, and to curvature estimates. It will be proved in Section 3

Proposition 2.1. Suppose that there is a finite collection of parametrizations $\Psi$ as in (9) whose ranges cover $K$ and which for some number $C_{0}$ satisfy the inequality

$$
r\left|\psi_{r}\right|<C_{0} .
$$

Also, suppose that there is a constant $C_{1}$ such that for the geodesic curvature $\kappa$ of $K_{r}$ in $K$ we have

$$
\int_{K_{r}}|\kappa| d \sigma<C_{1}
$$

where do denotes arc length.

Then there is a parametrization

$$
\Phi:(0, \varepsilon) \times S^{1} \rightarrow K
$$

satisfying (4) and (2), with $\delta \leq C_{0}\left(6+4 k\left(6+2 C_{1}\right)\right)$, where $k$ is the number of $\Psi$ needed to cover $K$. 
In Section 4, we prove that (11) is satisfied in our situation, with $C_{1}$ only depending on $V$ and $g$.

The following lemma shows that parametrizations $\Psi$ satisfying a sharper estimate than (10) exist for certain model families of curves in $\mathbb{R}^{2}$.

Lemma 2.2. Let $a, b$ be nonnegative integers, not both zero, and let $A>0$. There is a parametrization $\Psi(r, s)=(x(r, s), y(r, s))$ of each connected component of the family of curves

$$
x^{a} y^{b}=r, \quad r \in(0,1), \quad|x|+|y|<A,
$$

that satisfies

$$
\left|\Psi_{r}\right| \leq r^{\frac{1}{a+b}-1} .
$$

Here, $|\cdot|$ denotes the Euclidean metric on $\mathbb{R}^{2}$.

Proof. If $a=0$, then we can take $\Psi(r, s)=\left(s, \pm r^{1 / b}\right)$, and the inequality is clearly satisfied. Similarly for $b=0$.

Now assume $a>0, b>0$. We look at the curves in the first quadrant only; the other components are treated analogously. The desired parametrization $\Psi=(x, y)$ is defined by setting $s=x-y$. Namely, considering $x, y$ as functions of $r, s$ through the equations $s=x-y, r=x^{a} y^{b}$, with $x, y>0$, one obtains, by differentiating these equations with respect to $r$,

$$
x_{r}=y_{r}=r^{-1}\left(\frac{a}{x}+\frac{b}{y}\right)^{-1} .
$$

By the harmonic-geometric mean inequality, this is bounded above by

$$
r^{-1}(a+b)^{-1}\left(x^{a} y^{b}\right)^{1 /(a+b)}=(a+b)^{-1} r^{\frac{1}{a+b}-1} .
$$

The lemma follows.

Note that the extra power $1 /(a+b)$ in Lemma 2.2 will yield the estimate (3).

In order to reduce the general case to this model, we now "blow up" $\mathbb{R}^{N}$ to resolve the singularity $p$ of $V$. That is, we find an analytic manifold $X^{\prime}$ and a proper analytic map $\pi: X^{\prime} \rightarrow B$, where $B$ is a small ball around $p$ containing no other singularities of $V$, so that

(a) $\pi$ is a diffeomorphism $X^{\prime} \backslash E \rightarrow B \backslash p$, where $E=\pi^{-1}(p)$, and

(b) the "strict transform" $V^{\prime}:=\overline{\pi^{-1}(V \backslash p)}$ is a smooth surface.

Such an (embedded) resolution always exists, see [22] or 3]. For our application, we need to know some additional properties of this resolution:

Lemma 2.3. The resolution $\pi: X^{\prime} \rightarrow B$ can be chosen so that, in addition to (a) and (b) above, the following two properties are satisfied:

(c) Near any point $q \in E \cap V^{\prime}$ there are coordinates $y_{1}, \ldots, y_{N}$ centered at $q$ such that, near $q$,

$$
V^{\prime}=\left\{y_{3}=\ldots=y_{N}=0\right\} \quad \text { and } \quad r^{\prime}:=\pi^{*} r=\left|\prod_{i=1}^{N} y_{i}^{a_{i}}\right|
$$

for some nonnegative integers $a_{i}$. Here $r$ denotes the distance function to $p$ on $B$.

(d) Let $h=r^{-2}\left(g-d r^{2}\right)$ be the semi-Riemannian metric on $B \backslash p$ obtained from the spherical part of $g$. Then there is a smooth semi-Riemannian metric $h^{\prime}$ on $X^{\prime}$ such that, on $X^{\prime} \backslash E$, one has $h^{\prime}=\pi^{*} h$. 
Remark. Note that $h$ does not extend continuously to $p \in B$. Thus the point of (d) is that, after pulling back to $X^{\prime}$, a smooth extension from $X^{\prime} \backslash E$ to all of $X^{\prime}$ exists.

Before we prove this lemma, we finish the proof of Theorem 1.2. By Propositions 2.1 and 4.1, we are done if we construct parametrizations $\Psi$ covering $V$ that satisfy the estimate (10) with $C_{0}=C \varepsilon^{\alpha}$. To do this, we first find parametrizations $\Psi^{\prime}$ of $V^{\prime}$ and then push them down to $V$.

For each point $q \in V_{0}^{\prime}:=V^{\prime} \cap E$, let $U_{q}$ be a neighborhood of $q$ such that there are coordinates $y_{1}, \ldots, y_{N}$ on $U_{q}$ as in (c) above. Construct a parametrization $\Psi_{q}^{\prime}$ of each connected component of $V^{\prime} \cap U_{q} \cap\left\{r^{\prime}>0\right\}$ as follows: If this set is nonempty, then $a_{3}=\ldots=a_{N}=0$. Thus, $V^{\prime}$ is identified with the $\left(y_{1}, y_{2}\right)$-plane near zero, and $r^{\prime}=y_{1}^{a_{1}} y_{2}^{a_{2}}$. Let $\Psi_{q}^{\prime}$ be the parametrizations given by Lemma 2.2 The analogue of estimate (10), viz. $r\left|\left(\Psi_{q}^{\prime}\right)_{r}\right|_{k}<C_{0}$, is then satisfied for $k$ the Euclidean metric in the $\left(y_{1}, y_{2}\right)$-plane and therefore also for $k$ the smooth semi-Riemannian metric $h^{\prime}$, with

$$
C_{0}=C \varepsilon^{1 /\left(a_{1}+a_{2}\right)} \quad \text { for } r<\varepsilon .
$$

Here, $C$ is a constant only depending on $V$ (stemming from the length distortion between the Euclidean metric and the metric $h^{\prime}$ on $U_{q}$, i.e., $h^{\prime} \leq C g_{\text {eucl }}$ on $U_{q}$ ).

Fix $q$ and set $\Psi=\pi \circ \Psi_{q}^{\prime}$. By construction, $\Psi$ is of the form (9). We claim that $\psi$ satisfies (10), with $C_{0}$ as above. Since $\left(P C^{-1} \Psi\right)_{r}=\left(1, \psi_{r}\right)$, we have $\left|\Psi_{r}\right|_{h}=$ $\left|\left(P C^{-1} \Psi\right)_{r}\right|_{g_{S^{N-1}}}=\left|\psi_{r}\right|_{g_{S^{N-1}}}$. Therefore, we get $r\left|\psi_{r}\right|_{g_{S^{N-1}}}=r\left|\Psi_{r}\right|_{h}=r\left|\Psi_{q r}^{\prime}\right|_{h^{\prime}} \leq$ $C_{0}$, which was to be shown. Finally, since $r^{\prime}$ is proper, $V_{0}^{\prime}$ is compact. So finitely many of the $U_{q}$ cover $V_{0}^{\prime}$, and since $r^{\prime}$ must assume a positive minimum on the complement of their union $U$, there is an $\varepsilon_{0}>0$ such that $\left\{0<r^{\prime}<\varepsilon_{0}\right\}$ is contained in $U$. Thus, the assumptions of Proposition 2.1 are satisfied. So Theorem 1.2 is proved, with

$$
\alpha=\min \frac{1}{a_{1}+a_{2}},
$$

the minimum being taken over the finitely many coordinate neighborhoods $U_{q}$.

Proof of Lemma 2.3. First, we need a few definitions. Let $X$ be a real analytic manifold. A normal crossings divisor (n.c.d.) on $X$ is a subset $E \subset X$ which, near any $q \in X$, is a union of coordinate hyperplanes, in a suitable local coordinate system near $q$. A set $D$ has normal crossings with $E$ if the coordinates can be chosen so that, in addition, $D$ is a coordinate subspace. Finally, if $f$ is a smooth function on $X$, we say that $f$ has product form with respect to $E$ (and $D$ ) if $\{f=0\} \subset E$ and in one (and therefore any) such local coordinate system $y_{1}, \ldots, y_{N}$ near $q$ with $y(q)=0$, one has the representation

$$
f(y)=h(y) \prod_{i=1}^{N} y_{i}^{a_{i}}
$$

with $h$ smooth and $h(0) \neq 0$, and nonnegative integers $a_{i}$.

According to [3, a resolution can be obtained in the following way. One finds a sequence

$$
\begin{array}{lllllll}
X_{M} \stackrel{\pi_{M-1}}{\rightarrow} & X_{M-1} \\
E_{M} & & \rightarrow \ldots \rightarrow & X_{1} \stackrel{\pi_{0}}{\rightarrow} & X_{0}=B \\
E_{M-1} & & & E_{1}=\emptyset
\end{array}
$$

of spaces $X_{j}$, n.c.d.s $E_{j} \subset X_{j}$, and proper maps $\pi_{j}: X_{j+1} \rightarrow X_{j}$ as follows. Let $X_{0}=B, E_{0}=\emptyset$. If $X_{j}, E_{j}$ are defined, choose a suitable submanifold $D_{j} \subset X_{j}$ that 
is normal crossings with $E_{j}$, and let $X_{j+1}$ be the elementary blow-up of $X_{j}$ along $D_{j}$; that is, essentially $X_{j+1}$ is obtained from $X_{j}$ by replacing $D_{j}$ by its projectivized normal bundle, and $\pi_{j}$ is the obvious projection map; also, set $E_{j+1}=\pi_{j}^{-1}\left(D_{j} \cup E_{j}\right)$, which is normal crossings again. It is one of the main theorems in [22] and [3] that the $D_{j}$ can always be found such that after a finite number of such blow-ups, $X^{\prime}:=X_{M}$ is a resolution in the sense of (a) and (b) above, and such that $V^{\prime}$ and $E=E_{M}$ are normal crossings. Here, $\pi=\pi_{(M)}$, where $\pi_{(j)}=\pi_{0} \circ \ldots \circ \pi_{j-1}$. Also, one may choose $D_{j}$ to lie in the singular set of $\overline{\pi_{(j)}^{-1}(V \backslash p)}$ (of $V$ for $j=0$ ).

Assume first that $V$ is singular at $p$. We claim that (c) and (d) are satisfied for this resolution. The essential fact is that $V \cap B$ is only singular at $p$, so that $\pi_{0}: X_{1} \rightarrow B$ is just the blow-up of $p$. If we identify $B$ with a subset of $\mathbb{R}^{N}$ via $x \equiv t \omega$ for $x=P C(t, \omega)$, then this can be described as $X_{1}=\left(-\varepsilon_{0}, \varepsilon_{0}\right) \times S^{N-1} / \sim$, where $(t, \omega) \sim(-t,-\omega)$, and $\pi([t, \omega])=t \omega$. Then $E_{1}=\pi_{0}^{-1}(0)=\{t=0\}$. To prove (c), we show inductively that $R_{j}:=\pi_{(j)}^{*} r^{2}$ has product form with respect to $E_{j}$, for $j \geq 1$. This is clearly true for $j=1$, since $\pi_{0}^{*} r^{2}=t^{2}$ in the notation above. Then it follows directly from the definition of elementary blow-up that, if $R_{j}$ has product form with respect to $E_{j}$ and $D_{j}$ has normal crossings with $E_{j}$, then $R_{j+1}=\pi_{j}^{*} R_{j}$ has product form with respect to $E_{j+1}$. (Use projective coordinates near points in $\pi_{j}^{-1}\left(D_{j}\right)$.) Therefore, $R_{M}=\pi^{*} r^{2}$ has the form (12). Since $R_{M} \geq 0$, $h$ is positive and all $a_{i}$ are even. Since $E=\left\{R_{M}=0\right\}$, one of the $a_{i}$ is nonzero for $q \in E$; so one may modify the coordinates so that $h \equiv 1$. This proves (c).

To prove (d), we note that the pull-back metric on $\left(-\varepsilon_{0}, \varepsilon_{0}\right) \times S^{N-1}$ is $d t^{2}+$ $t^{2} g_{S^{N-1}}(t)$ with $g_{S^{N-1}}(t)$ as in (8) for $t \geq 0$ and $g_{S^{N-1}}(-t)=A^{*} g_{S^{N-1}}(t)$, where $A$ : $\omega \mapsto-\omega$ is the antipodal map. This shows that $h_{1}:=g_{S^{N-1}}(t)$ is well-defined and smooth on $X_{1}$ and satisfies $h_{1}=\pi_{0}^{*} h$ on $X_{1} \backslash E_{1}$. Therefore, $h^{\prime}=\left(\pi_{1} \circ \ldots \circ \pi_{r-1}\right)^{*} h_{1}$ is also smooth.

Finally, if $V$ is smooth at $p$, then just blow up $p$ once; then (c) and (d) are satisfied by the same argument.

\section{BASICS ON QUASI-ISOMETRY}

In this section we prove Proposition 2.1

Lemma 3.1. Let $\Psi$ be a local parametrization as in (9). Then

$$
\Psi^{*} g \simeq d r^{2}+H(r, s)^{2} d s^{2}
$$

for some function $H$ if and only if

$$
r\left|\psi_{r}(r, s)\right|<C
$$

for some constant $C$. In this case, one can take $H=r\left|\psi_{s}\right|$. Then the quasi-isometry constant depends only on $C$, and for $C<1 / 2$ it can be taken to be $1+2 C$.

Proof. From (8) and $\Psi=P C \circ(r, \psi)$ we have

$$
\begin{aligned}
\Psi^{*} g & =d r^{2}+r^{2}|d \psi|^{2} \\
& =d r^{2}+r^{2}\left|\psi_{r} d r+\psi_{s} d s\right|^{2} \\
& =\left(1+|A|^{2}\right) d r^{2}+2 A \cdot B d r d s+|B|^{2} d s^{2},
\end{aligned}
$$

where $A=r \psi_{r}, B=r \psi_{s}$. We now use the following elementary lemma. 
Lemma 3.2. Let $g=a d x^{2}+2 b d x d y+c d y^{2}$ be a positive definite quadratic form, and let $\tilde{g}=a d x^{2}+c d y^{2}$ be its "diagonal part". Set

$$
T=\frac{|b|}{\sqrt{a c}} \text {. }
$$

Then one has

$$
(1-T) \tilde{g} \leq g \leq(1+T) \tilde{g},
$$

and these inequalities are sharp.

Remark. Note that $T$ is the cosine of the angle between the $x$-axis and the $y$-axis with respect to the Riemannian metric $g$ on $\mathbb{R}^{2}$. Therefore, if $a, b, c$ are allowed to depend on $x$ and $y$, one obtains that $g \approx \tilde{g}$ if and only if this angle is bounded away from zero, and the quasi-isometry constant is close to one if this angle is close to $\pi / 2$.

Proof. The left inequality is equivalent to $T a d x^{2}+2 b d x d y+T c d y^{2} \geq 0$, and this is equivalent to $T a \geq 0$ and $T a \cdot T c-b^{2} \geq 0$. Similarly for the right inequality. This implies the claim. Note that always $T<1$, since $g>0$ implies $a c-b^{2}>0$.

End of proof of Lemma 3.1. Here we have, for fixed $(r, s)$, that $a=1+|A|^{2}, b=$ $A \cdot B$ and $c=|B|^{2}$; so

$$
T=\frac{|A \cdot B|}{|B| \sqrt{1+|A|^{2}}} \leq \frac{|A|}{\sqrt{1+|A|^{2}}} .
$$

Also, one clearly has $d r^{2}+|B|^{2} d s^{2} \approx_{1+|A|^{2}}\left(1+|A|^{2}\right) d r^{2}+|B|^{2} d s^{2}$. Therefore, from Lemma 3.2 we get a quasi-isometry factor, for fixed $(r, s)$, of at most

$$
\left(1+|A|^{2}\right)\left(1-\frac{|A|}{\sqrt{1+|A|^{2}}}\right)^{-1} .
$$

An easy calculation shows that this is bounded for $A$ bounded, and for $|A|<1 / 2$ it is bounded above by $1+2 A$. Finally, looking at the length of $\partial / \partial r$, one sees that $|A|<C$ is also necessary for the asserted quasi-isometry.

The following lemma relates arbitrary parametrizations to (unrenormalized) arc length parametrizations.

Lemma 3.3. Let $\Omega(r, t)=P C(r, \omega(r, t)),(r, t) \in\left(r_{1}, r_{2}\right) \times\left(t_{1}, t_{2}\right)$ be a local parametrization of $K$ with $\omega$ arc length on the sphere, i.e., $\left|\omega_{t}\right| \equiv 1$. Assume that there is a parametrization $\Psi:(0, \varepsilon) \times(-A, A) \rightarrow K$ whose range contains the range of $\Omega$ and which satisfies the inequality (10), and that (11) holds.

Then, if $r\left|\omega_{r}(r, t)\right|<C$ holds for one value $t=t_{0}$, it holds for all $t$ (with $C$ replaced by $C+C_{0} C_{2}$, where $C_{2}=6+2 C_{1}$ ).

Proof. We may assume that $\Psi$ and $\Omega$ trace the lines $r=$ const in the same direction. Define the function $b(r)$ by the equation

$$
\omega\left(r, t_{0}\right)=\psi(r, b(r)) .
$$

Then, if we set $l(r, s)=\int_{b(r)}^{s}\left|\psi_{s}(r, \sigma)\right| d \sigma$, we must have

$$
\omega\left(r, t_{0}+l(r, s)\right)=\psi(r, s) .
$$

Differentiating in $r$, we get $\omega_{r}+l_{r} \omega_{t}=\psi_{r}$, evaluated at corresponding points. From condition (10) and $\left|\omega_{t}\right|=1$ we get

$$
r\left|\omega_{r}\left(r, t_{0}+l(r, s)\right)\right|<C \Leftrightarrow r\left|l_{r}(r, s)\right|<C,
$$


where $C$ must be replaced by $C+C_{0}$ in either direction of the implication. Now we have $l_{r}=a(r)+\int_{b(r)}^{s} \frac{\partial}{\partial r}\left|\psi_{s}\right| d s$, where $a(r)=-b^{\prime}(r)\left|\psi_{s}(r, b(r))\right|$. The variation of length formula says that

$$
\int_{b(r)}^{s} \frac{\partial}{\partial r}\left|\psi_{s}\right| d s=\left.\left(\frac{\psi_{s}}{\left|\psi_{s}\right|}, \psi_{r}\right)\right|_{b(r)} ^{s}-\int_{b(r)}^{s}\left(N, \psi_{r}\right) \kappa d \sigma
$$

where $\kappa$ is the geodesic curvature, $N$ is the unit normal, and $\sigma$ denotes arc length. From (10) and (11) we get $r\left|l_{r}-a\right| \leq C_{0}\left(2+C_{1}\right)$. Therefore, the right side of (14) is equivalent to $r|a(r)|<C$, where the constant gets worse by $C_{0}\left(2+C_{1}\right)$. Now since this last condition is independent of $s$, and since the left side of (14) is true for $s=b(r)$ by assumption, it must be true for all $s$. Altogether we lose at most $C_{0}\left(6+2 C_{1}\right)$ in the constant.

Proof of Proposition 2.1. First we consider a parametrization

$$
\begin{aligned}
\Omega:(0, \varepsilon) \times[0, \infty) & \rightarrow K, \\
(r, t) & \mapsto P C(r, \omega(r, t)),
\end{aligned}
$$

for which $\omega$ is arc length on the sphere, i.e., $\left|\omega_{t}\right| \equiv 1$. Such a parametrization is determined when the "initial" curve $r \mapsto \Omega(r, 0)$ and an orientation for the family of curves $K_{r}$ is prescribed. We assume that such an orientation is given and prescribe the initial curve by choosing any one local parametrization $\psi$ and setting

$$
\omega(r, 0)=\psi(r, 0) .
$$

Note that $\left|\Omega_{t}\right|=r$ and length $\left(K_{r}\right)=l(r)$ imply that

$$
\omega(r, \tilde{l}(r))=\omega(r, 0)
$$

for $\tilde{l}(r)=l(r) / r$. We now prove that the parametrization $\omega$ thus defined satisfies the inequality

$$
r\left|\omega_{r}(r, t)\right|<C
$$

for $t \leq \tilde{l}(r)$, with $C=\left(1+k C_{2}\right) C_{0}$. Fix $r_{0} \in(0, \varepsilon)$. We prove (17) for $r=r_{0}$, but with $C$ independent of $r_{0}$. Since each of the finitely many given parametrizations $\Psi$ covers an interval on the curve $\gamma=K_{r_{0}}$, one can choose points $p_{0}=$ $\Omega\left(r_{0}, 0\right), p_{1}, p_{2}, \ldots, p_{k}=p_{0}$ in this order on $\gamma$ such that each subarc from $p_{i}$ to $p_{i+1}$ of $\gamma$ is contained in the range of some parametrization $\Psi_{i}$ satisfying the estimates stated in the proposition. Define $0=t_{0}<t_{1}<\ldots<t_{k}=\tilde{l}\left(r_{0}\right)$ by letting $r t_{i}$ (for $i<k$ ) be the distance from $p_{0}$ to $p_{i}$ measured along $\gamma$ in the positive direction; then $\Omega\left(r_{0}, t_{i}\right)=p_{i}$. We prove by induction on $i$ that (17) is true for $t \leq t_{i}$. For $i=0$ we have $t_{0}=0$ and therefore (17) is true by (15), with $C=C_{0}$. Suppose it is true for $t \leq t_{i}$ with some constant $C=C_{(i)}$. Since the range of $\Psi_{i}$ is open, it will contain the range of $\Omega$ when restricted to a small interval around $r_{0}$ times some interval containing $\left[t_{i}, t_{i+1}\right]$. Thus Lemma 3.3 implies that (17) holds for $t \in\left[t_{i}, t_{i+1}\right]$, with $C=C_{(i+1)}=C_{(i)}+C_{2} C_{0}$. Altogether, we get that (17) holds for all $t \leq \tilde{l}(r)$ with $\left.C=\left(1+k C_{2}\right)\right) C_{0}$.

Now we renormalize $\omega$ by setting

$$
\phi(r, \theta)=\omega(r, \theta \tilde{l}(r) /(2 \pi)) .
$$

We show that $\phi$ also satisfies the inequality

$$
r\left|\phi_{r}(r, \theta)\right|<C,
$$


where now $C=\left(3+2 k C_{2}\right) C_{0}$ and $\theta \in[0,2 \pi]$. Differentiating (16) in $r$, we get

$$
\omega_{r}(r, 0)=\omega_{r}(r, \tilde{l}(r))+\tilde{l}^{\prime}(r) \omega_{t}(r, \tilde{l}(r)) .
$$

Now (17) and $\left|\omega_{t}\right|=1$ give $r\left|\tilde{l}^{\prime}\right|<\left(2+k C_{2}\right) C_{0}$, and by (18) this in turn implies (19).

Now set $\Phi=P C \circ(r, \phi)$. By definition, we have $\left|\Phi_{\theta}\right|=r\left|\phi_{\theta}\right|=r \tilde{l}(r) /(2 \pi)=$ $l(r) /(2 \pi)$, and Lemma 3.1 applied to $\Phi$, gives (2).

\section{UNIFORM INTEGRABILITY OF GEODESIC CURVATURE}

Here we prove that the bound (11) is satisfied in our situation.

Proposition 4.1. Let $V, g, K, K_{r}$ be as in the statement of Theorem 1.2, and denote by $\kappa$ the geodesic curvature of the curves $K_{r}$ in $V$, and by $d \sigma$ arc length. Then

$$
\int_{K_{r}}|\kappa| d \sigma<C_{1}
$$

for $r<\varepsilon_{0}$, with $C_{1}$ only depending on $V$ and $g$.

Proof. Let $N K_{r}$ denote the unit normal bundle of $K_{r}$ in $V$, a one-dimensional submanifold of the unit tangent bundle of $V_{0}$. It is an elementary fact from differential geometry that

$$
\int_{K_{r}}|\kappa| \leq \operatorname{length}\left(N K_{r}\right) .
$$

So it remains to show that these lengths are uniformly bounded as $r \rightarrow 0$. Set $U=$ $\bigcup_{r \in(0, \varepsilon)} N K_{r}$. Now $U$ is a semi-analytic subset of $T^{1} \mathbb{R}^{N}$, the unit tangent bundle of $\left(\mathbb{R}^{N}, g\right)$. This can be seen as follows. Assume $V$ is given as $\left\{f_{1}=\ldots=f_{L}=0\right\}$ near $p$. If $V$ is a complete intersection at $p$, i.e., $L=N-2$, then we can write

$$
U=\left\{(x, v) \in T^{1} \mathbb{R}^{N}: d f_{i \mid x}(v)=0, \omega_{x}(v)=0, x \in K \backslash p\right\} ;
$$

here the analytic one-form $\omega$ is defined as $\omega=*\left(d\left(r^{2}\right) \wedge d f_{1} \wedge \ldots \wedge d f_{L}\right)$, where * denotes the Hodge star operator. If $V$ is not a complete intersection at $p$, then define, for every subset $S \subset\{1, \ldots, L\}$ with $N-2$ elements, the one-form

$$
\omega_{S}=*\left(d\left(r^{2}\right) \wedge \bigwedge_{i \in S} d f_{i}\right)
$$

and use the conjunction of $\omega_{S \mid x}(v)=0$ over all $S$ in the description of $U$.

We now have the following situation: $X=T^{1} \mathbb{R}^{N}$ is a Riemannian real analytic manifold, with metric induced from the given metric on $\mathbb{R}^{N} . U \subset X$ is a twodimensional semi-analytic subset and $R:=r^{2}: X \rightarrow \mathbb{R}$ is a proper analytic function that is non-constant on $U$. We claim that this implies that the level sets of $R$ have bounded lengths (i.e., one-dimensional Hausdorff measure), for bounded $R$. This can be proved as follows (see 4] for definitions and theorems used). $\bar{U}$ is semianalytic and two-dimensional. Let $\pi: Y \rightarrow X$ be a uniformization of $\bar{U}$; this means that $Y$ is smooth and two-dimensional, $\pi$ is proper, and $\pi(Y)=\bar{U}$. (A uniformization can be obtained from a resolution, but also in a simpler way, see 4].) Let $h$ be the pull-back semi-Riemannian metric on $Y$. Now $R^{\prime}=\pi^{*} R: Y \rightarrow \mathbb{R}$ is proper. Clearly, the length of any level line $\{R=t\}$ in $X$ is bounded by the length (with respect to $h$ ) of the level line $\left\{R^{\prime}=t\right\}$ in $Y$. Therefore, we are now in the smooth two-dimensional case, which is easy. For example, one can blow up (fairly explicitly) $Y$ to put $R^{\prime}$ into local product form; then the level sets are 
families of hyperbolas locally, and since the pull-back semi-metric is smooth, they have uniformly bounded length (compare Section 5).

\section{Asymptotics of $l(r)$}

We investigate $l(r)$ by evaluating it on the blown-up space $X^{\prime}$, described in Section 2 The pull-back metric then degenerates on the preimage of $\{r=0\}$.

We first analyze the model case, where the curves are given in $\mathbb{R}^{2}$ by $x^{a} y^{b}=r$, but the metric may degenerate on the coordinate axes.

Proposition 5.1. Let $a, b$ be nonnegative integers, $a+b>0$. Let $h$ be a real analytic semi-Riemannian metric defined near zero in $\mathbb{R}^{2}$ that is Riemannian for $x^{a} y^{b} \neq 0$, and $\chi \in C_{0}^{\infty}\left(\mathbb{R}^{2}\right)$ a cutoff function that is nonnegative and equals one near the origin. Let $l_{\chi}(r)=l_{\chi, h}(r)$ be the length of the curve $x^{a} y^{b}=r$, weighted by $\chi$, i.e.,

$$
l_{\chi}(r)=\int\left|\psi_{s}(r, s)\right|_{h_{\psi(r, s)}} \chi(\psi(r, s)) d s
$$

for some parametrization $\psi$ of this family of curves.

Then $l_{\chi}(r)$ has an asymptotic expansion, as $r \rightarrow 0$,

$$
l_{\chi}(r) \sim \sum_{i, j} C_{i, j} r^{i}(\log r)^{j},
$$

where $i$ ranges over $\frac{1}{m} \mathbb{N}_{0}$ for some fixed $m \in \mathbb{N}$, and $j \in\{0,1\}$. This expansion may be differentiated (arbitrarily often) term by term.

If, in addition, $h$ is the pull-back of a nondegenerate (i.e., Riemannian) metric under an analytic map that has injective differential for $x^{a} y^{b} \neq 0$, then the leading term is of the form $r^{\gamma}$.

Note. The following example shows that, without further assumptions, $\log r$ may appear in the leading term. Let $h=y^{2} d x^{2}+x^{2} d y^{2}$ and $a=b=1$. Then, parametrizing by $s=x$, one has

$$
\left|\psi_{s}\right|_{h}=\left|\left(1,-r / x^{2}\right)\right|_{h}=\left((r / x)^{2}+x^{2}\left(-r / x^{2}\right)\right)^{1 / 2}=\sqrt{2} r / x
$$

which is easily seen to imply $l_{\chi}(r) \sim-\sqrt{2} r \log r$.

Proof. Clearly, $l_{\chi}$ is bounded, smooth for $r>0$, and has compact support. We use the Mellin transform, defined for a bounded compactly supported function $l$ by

$$
(M l)(z)=\int_{0}^{\infty} r^{z} l(r) \frac{d r}{r}
$$

with $z \in \mathbb{C}$. This integral is defined and holomorphic for $\operatorname{Re}(z)>0$. As is wellknown (see 24], for example), an asymptotic expansion for $l$ as $r \rightarrow 0$ (with derivatives) is equivalent to the existence of a meromorphic continuation of $M l(z)$ to the whole $z$-plane, with a pole of order $\mu$ at $z_{0}$ corresponding to a term $r^{-z_{0}}(\log r)^{\mu-1}$ in the asymptotics, and such that one has decay for large imaginary part, i.e.,

$$
|M l(z)| \leq C_{N}|\operatorname{Im} z|^{-N}
$$

for any $N$, uniformly for bounded $\operatorname{Re} z$ and away from the poles. Therefore, we have to show the existence of such a continuation with at most double poles, at points in $-\mathbb{N}_{0} / m$. The additional statement on the leading term will be proved directly.

We assume $a>0, b>0$; the other case is only slightly different, but easier. 
A simple calculation, for example using the parameter $s=x$, shows that, as measures,

$$
\left|\psi_{s}\right|_{h_{\psi}} d s \frac{d r}{r}=|(b x,-a y)|_{h_{(x, y)}} \frac{d x}{x} \frac{d y}{y} .
$$

Therefore, we get

$$
M l_{\chi}(z)=\int_{0}^{\infty} \int_{0}^{\infty} x^{a z} y^{b z} p(x, y)^{1 / 2} \chi(x, y) \frac{d x}{x} \frac{d y}{y},
$$

where we have used $r=x^{a} y^{b}$, and where the analytic function $p$ is defined as $p(x, y)=|(b x,-a y)|_{h_{(x, y)}}^{2}$. If we set $x=x_{1}^{2}, y=x_{2}^{2}$, then (21) becomes

$$
M l_{\chi}(z)=\int_{-\infty}^{\infty} \int_{-\infty}^{\infty}\left|x_{1}\right|^{2 a z-1}\left|x_{2}\right|^{2 b z-1} p\left(x_{1}^{2}, x_{2}^{2}\right)^{1 / 2} \chi\left(x_{1}^{2}, x_{2}^{2}\right) d x_{1} d x_{2},
$$

and the asymptotics follows from the following proposition:

Proposition 5.2. Let $p, q$ be real analytic functions defined in a neighborhood $U$ of the support of a function $\rho \in C_{0}^{\infty}\left(\mathbb{R}^{n}\right)$. Let $A, B \in \mathbb{R}, B \geq 0$. For $z \in \mathbb{C}$, $\operatorname{Re} z \geq-A$, define

$$
f(z)=\int_{\mathbb{R}^{n}}|q(x)|^{z+A}|p(x)|^{B} \rho(x) d x .
$$

Then

(a) $f$ can be extended meromorphically to all of $\mathbb{C}$, with poles of order at most $n$.

(b) If, for every $x \in \operatorname{supp} \rho, q(x) \neq 0$ implies $p(x) \neq 0$ and $d q_{\mid x} \neq 0$, then

$$
|f(z)| \leq C_{N}|\operatorname{Im} z|^{-N}
$$

for any $N$, uniformly in $|\operatorname{Im} z| \geq 1$ and for bounded $\operatorname{Re} z$.

Proof. Part (a) is a consequence of a more general theorem by Gel'fand and Bernstein [2]. We sketch their proof and show how one obtains (b). First, we use the resolution of singularities theorem to find an analytic manifold $X$ and an analytic proper map $\pi: X \rightarrow U$ so that (1) $\pi$ is a diffeomorphism outside $\{p q=0\}$ and (2) the pull-back $\pi^{*}(p q)$ has local product form; i.e., near any point in $X$ there are analytic local coordinates $\left(y_{1}, \ldots, y_{n}\right)$ centered at that point such that $\pi^{*}(p q)$ is a monomial in the $y_{i}$ times a non-vanishing analytic function. Then $\pi^{*} p$ and $\pi^{*} q$ must have local product form, too, and if we write $\pi^{*}\left(d x_{1} \wedge \ldots \wedge d x_{n}\right)=S(y) d y_{1} \wedge \ldots \wedge d y_{n}$, then $S$ must have local product form (this follows from Hilbert's Nullstellensatz resp. Rückert's Nullstellensatz in the analytic case, see [28] - since $S$ vanishes only where $\pi^{*}(p q)$ vanishes, even after local complexification). All this implies that we can use a partition of unity on $X$ to write $f$ as a finite sum of terms of the form

$$
g(z)=\int_{\mathbb{R}^{n}}|\tilde{q}(y)|^{z+A}|\tilde{p}(y)|^{B}|S(y)| \tilde{\rho}(y) d y,
$$

where $\tilde{\rho} \in C_{0}^{\infty}\left(\mathbb{R}^{n}\right), \tilde{q}(y)=\pi^{*} q(y)=y^{\alpha} h_{1}(y), \tilde{p}(y)=\pi^{*} p(y)=y^{\beta} h_{2}(y), S(y)=$ $y^{\gamma} h_{3}(y)$ with multi-indices $\alpha, \beta, \gamma$ and $h_{1}, h_{2}, h_{3}$ non-vanishing on supp $\tilde{\rho}$. For each $g$ we have one of the following two cases:

(I) $\alpha \neq 0$, i.e., $\tilde{q}(0)=0$.

(II) $\alpha=0$; then $\gamma=0$ by (1) above, and under the additional condition in part 
(b) of the proposition also $\beta=0, d \tilde{q} \neq 0$.

We first consider case (II). If $\alpha=0$, then $|\tilde{q}|^{z}$ and therefore $g$ is entire in $z$, which proves (a). For (b), we may assume w.l.o.g. that $\tilde{q}(y)=e^{y_{1}}$; then

$$
g(R+i I)=\int_{-\infty}^{\infty} e^{i I y_{1}}\left(e^{(R+A) y_{1}} \int_{\mathbb{R}^{n-1}}|\tilde{p}|^{B}|S| \tilde{\rho} d y^{\prime}\right) d y_{1} .
$$

Since $\tilde{p}, S \neq 0$ on supp $\tilde{\rho}$, this is the Fourier transform of a smooth compactly supported function, therefore rapidly decreasing in $I$.

We now consider case (I). By a smooth change of coordinates, we may arrange $h_{1} \equiv 1$. Then (with $\left.\delta=\alpha A+\beta B+\gamma\right)$

$$
g(z)=\int_{\mathbb{R}^{n}}\left|y_{1}\right|^{\alpha_{1} z+\delta_{1}} \cdots\left|y_{n}\right|^{\alpha_{n} z+\delta_{n}} \chi(y) d y, \quad \chi \in C_{0}^{\infty}\left(\mathbb{R}^{n}\right) .
$$

The following stronger result now implies the proposition, with $m$ equal to the least common multiple of all the positive $a_{i}$, over all $i$ and $g$ : If $\chi \in C_{0}^{\infty}\left(\mathbb{R}^{n}\right)$, then the function

$$
g\left(z_{1}, \ldots, z_{n}\right)=\int_{\mathbb{R}^{n}}\left|y_{1}\right|^{z_{1}} \cdots\left|y_{n}\right|^{z_{n}} \chi(y) d y
$$

has a meromorphic continuation from $\left\{\operatorname{Re} z_{i} \geq 0 \forall i\right\}$ to $\mathbb{C}^{n}$, with at most simple poles on the hyperplanes $\left\{z_{i}=-s\right\}, s \in \mathbb{N}$, and on $\left\{\left(z_{1}, \ldots, z_{n}\right): \operatorname{dist}\left(z_{i},-\mathbb{N}\right)>\right.$ $\left.\varepsilon,\left|\operatorname{Re} z_{i}\right|<\varepsilon^{-1} \forall i\right\}$ one has for any $N$

$$
\left|g\left(z_{1}, \ldots, z_{n}\right)\right| \leq C_{\varepsilon, N} \prod_{i=1}^{n}\left|\operatorname{Im} z_{i}\right|^{-N}
$$

where $C_{N}$ is bounded in terms of the $C^{M}$ norm of $\chi$ for some $M=M(N)$. This claim follows for $n=1$ directly from standard facts about distributions, see [19], and for $n>1$ by induction.

It remains to prove the additional statement in Proposition 5.1 about the leading term. Assume $h=\pi^{*} g$ for an analytic map $\pi:\left(\mathbb{R}^{2}, 0\right) \rightarrow\left(\mathbb{R}^{N}, 0\right)$ as in the proposition and a Riemannian metric $g$ on $\mathbb{R}^{N}$ (everything defined near zero). Then $\left|\psi_{s}\right|_{h}=\left|\partial_{s}(\pi \circ \psi)\right|_{g}$. Assume $b>0$. We will use the parametrization by $s=x$ now, i.e.,

$$
\psi(r, s)=\left(s, r^{1 / b} / s^{a / b}\right) .
$$

A short calculation (as above) shows that

$$
\partial_{s}(\pi(\psi(r, s)))=\frac{1}{b x}\left(b x \pi_{x}-a y \pi_{y}\right) \in \mathbb{R}^{N},
$$

where $(x, y)=\psi(r, s)$. Now the essential fact is the following: As a function of $r$ and $s$, every component of (22) is a Laurent series in $s^{1 / b}$ whose coefficients are power series in $r^{1 / b}$. Since it is a derivative in $s$, the $s$-residue, i.e., the coefficient of $s^{-1}$, vanishes. This means that no component of the analytic function $b x \pi_{x}-a y \pi_{y}$ contains a monomial of the form $\left(x^{a} y^{b}\right)^{\alpha}$. Also, this function does not vanish identically by the nondegeneracy hypothesis on $\pi$. In particular, letting $\gamma \in \mathbb{Q}$ be the largest number such that $\left(x^{a} y^{b}\right)^{\gamma}$ divides $b x \pi_{x}-a y \pi_{y}$, we have

$$
b x \pi_{x}-a y \pi_{y}=\left(x^{a} y^{b}\right)^{\gamma} Q(x, y)
$$

with $Q: \mathbb{R}^{2} \rightarrow \mathbb{R}^{N}$ analytic in certain fractional powers of $x$ and $y$ and $Q(0,0)=0$, and such that $Q$ does not vanish identically on $\{x=0\} \cup\{y=0\}$. Since $g$ is nondegenerate, $q=|Q|_{g}^{2}$ has the same properties. 
To finish the proof, it is clearly enough to show that, for some constants $0<c<$ $C<\infty$, we have

$$
c r^{\gamma}<l_{\chi}(r)<C r^{\gamma},
$$

for small $r$. From (22) and the definition of $q$, we have

$$
l_{\chi}(r)=r^{\gamma} I(r) / b, \quad \text { where } I(r)=\int q(x, y)^{1 / 2} \chi(x, y) \frac{d x}{x},
$$

where always $y=r^{1 / b} / x^{a / b}$. To show $I<C$, we observe that $q(0,0)=0$ implies $q(x, y) \leq x^{B}+y^{B}$ for some $B>0$. Since $\frac{d x}{x}=\frac{b}{a} \frac{d y}{y}$, we have

$$
I \leq \int_{0}^{1} x^{B} d x / x+b / a \int_{0}^{1} y^{B} d y / y<C .
$$

To show $I>c$, we recall that $q$ does not vanish identically on at least one coordinate axis, say the $x$-axis. Then, $q^{1 / 2}>c_{0}>0$ on some rectangle $J \times[0, \delta]$ on which $\chi=1$, where $J$ is an open interval on the $x$-axis and $\delta>0$. Then $I \geq c_{0} \int_{J} d x / x=: c$ whenever $r$ is so small that $x^{a} y^{b}=r, x \in J$ implies $y<\delta$. This finishes the proof of the proposition.

Proof of Theorem 1.3. Apply the proposition to the semi-Riemannian metric $h_{1}=$ $\pi^{*} g$ and to $h_{2}=h^{\prime}$ from Lemma 2.3(d), and use a partition of unity on $X^{\prime}$. Clearly $l_{\chi, h_{1}}=r l_{\chi, h_{2}}$. Therefore, the leading term has exponent $\gamma \geq 1$. Also, since $g$ is nondegenerate, there is no logarithm.

\section{The Gauss-Bonnet Theorem}

Here we prove Theorem 1.4. The integrability of $\mathcal{K}$, i.e., the finiteness of $\int_{V_{0}}|\mathcal{K}|$, is proved in a similar way as the bound on geodesic curvature (11), only simpler. We sketch the argument in three dimensions; for details see [17. From the fact that $\mathcal{K}$ is the Jacobian of the differential of the Gauss map, one easily sees that for any open, relatively compact set $U \subset V_{0}$ one has $\int_{U}|\mathcal{K}| \leq \operatorname{area}(N U)$, where $N U$ is the unit normal bundle of $U$, with the induced metric. Since $V_{0}$ is semi-analytic, so is $N V_{0}$; so it has finite area by compactness of $V$. Therefore, $\int_{V_{0}}|\mathcal{K}|$ is finite also.

To prove the Gauss-Bonnet formula, we choose $\varepsilon_{0}>0$ small enough so that Theorem 1.2 applies for each $p_{i}$ (but here we only need the topological statement), and let $U_{\varepsilon}=\bigcup_{i=1}^{R} B_{\varepsilon}\left(p_{i}\right)$ and $V_{\varepsilon}=V \backslash U_{\varepsilon}$. For $\varepsilon<\varepsilon^{\prime}<\varepsilon_{0}$ we use the additivity of the Euler characteristic and $V=V_{\varepsilon} \cup U_{\varepsilon^{\prime}}$ to obtain

$$
\chi(V)=\chi\left(V_{\varepsilon}\right)+\chi\left(U_{\varepsilon^{\prime}}\right)-\chi\left(V_{\varepsilon} \cap U_{\varepsilon^{\prime}}\right) .
$$

Now $U_{\varepsilon^{\prime}}$ is homotopic to $\left\{p_{1}, \ldots, p_{R}\right\}$; so

$$
\chi\left(U_{\varepsilon^{\prime}}\right)=R,
$$

and $V_{\varepsilon} \cap U_{\varepsilon^{\prime}}$ is homotopic to a disjoint union of circles; so

$$
\chi\left(V_{\varepsilon} \cap U_{\varepsilon^{\prime}}\right)=0 .
$$

Applying the classical Gauss-Bonnet Theorem (see [29], Ch. 6) to the manifold with boundary $V_{\varepsilon}$, we get

$$
\chi\left(V_{\varepsilon}\right)=\frac{1}{2 \pi} \int_{V_{\varepsilon}} \mathcal{K}+\frac{1}{2 \pi} \int_{\partial V_{\varepsilon}} \kappa,
$$


where $\kappa$ is the geodesic curvature of $\partial V_{\varepsilon}=\partial U_{\varepsilon}$ with respect to the normal pointing outward from $V_{\varepsilon}$. Now the variation of arc length is geodesic curvature (see [29], Ch. 9), i.e., (with the previous orientation),

$$
\frac{d}{d \varepsilon} l\left(\partial V_{\varepsilon}\right)=-\int_{\partial V_{\varepsilon}}(\partial / \partial r, N) \kappa
$$

where $N$ is the unit normal (pointing away from the $p_{i}$ ) and $\partial / \partial r$ is taken with respect to the coordinates described in Theorem [1.2 Now this theorem implies that the scalar product $(\partial / \partial r, N)$ tends to 1 as $r=\varepsilon \rightarrow 0$, uniformly in $\theta$. Since $\int_{\partial V_{\varepsilon}}|\kappa|$ is uniformly bounded (Proposition 4.1), we get

$$
\frac{d}{d \varepsilon} l\left(\partial V_{\varepsilon}\right)=-\int_{\partial V_{\varepsilon}} \kappa+o(\varepsilon)
$$

By Theorem 1.3, we have

$$
\frac{d}{d \varepsilon} l\left(\partial V_{\varepsilon}\right)_{\mid \varepsilon=0}=\sum_{i=1}^{S} l_{i} .
$$

Therefore, taking the limit as $\varepsilon \rightarrow 0$ in (23) and (24), we get the result.

\section{Analysis near a Singular POINT}

Here we prove Theorem 1.5 For a differential operator $D$ on forms, denote by $D_{\min }$ and $D_{\max }$ the unbounded operators in $L^{2}:=L^{2}\left(\bigwedge V_{0}\right)$ with domains $\{\omega \in$ $L^{2}: \exists \omega_{n} \in C_{0}^{\infty}\left(V_{0}\right), \omega_{n} \rightarrow \omega$ and $D \omega_{n} \rightarrow D \omega$ in $\left.L^{2}\right\}$ and $\left\{\omega \in L^{2}: D \omega \in L^{2}\right\}$, respectively.

Proof of (i). First, we show that validity of the $L^{2}$ Stokes Theorem depends only on the quasi-isometry class of the metric. This is not obvious, since the local expression for $d^{t}$ contains derivatives of the metric coefficients. But it follows directly from the fact that (6) is equivalent to the statement

$$
d_{\min }=d_{\max }
$$

and the fact that these two operators (in particular, their domains) are quasiisometry invariants. To see this equivalence, note that (6) is equivalent to

$$
\left(d_{\max }\right)^{*}=\left(d^{t}\right)_{\max }
$$

where the star denotes the functional analytic adjoint. Also, it is easy to see that $\left(d_{\min }\right)^{*}=\left(d^{t}\right)_{\max }$. Since $d_{\min }$ and $d_{\max }$ are closed operators, the claim follows. For details, see [20].

By Theorem 1.1 it is therefore enough to prove (6) for metrics on $V_{0}$ that are exactly horn- or cone-like near the singularities. This is a special case of a wellknown result by Cheeger [14], Theorem 2.2.

Proof of (ii). A selfadjoint extension of $D_{G B}$ is given by $d_{\max }+\left(d^{t}\right)_{\max }$. This is selfadjoint by (25). Its domain is $\left\{\omega \in L^{2}: d \omega, d^{t} \omega \in L^{2}\right\}$. Then its square is a selfadjoint extension of $\Delta$. Discreteness of the spectrum is a quasi-isometry invariant by [9] (Lemma 2.17 and the remarks before (3.13)), and was proved for cones and horns by Cheeger [15] and Lesch and Peyerimhoff [25].

Proof of (iii). This statement is quasi-isometrically invariant, see 15, Lemma 7.1, and was proved for cones by Cheeger [15] and for horns by Brüning [8], 7] and Callias [13]. 
Proof of (iv). One can argue as in the proof of Theorem 1.4. $\chi_{(2)}$ is also additive. For manifolds with boundary (and metrics smooth up to the boundary) or their open interior, one has $\chi_{(2)}=\chi$. So only the term $\chi_{(2)}\left(U_{\varepsilon^{\prime}}\right)$ in (23) needs to be reevaluated. Now $\chi_{(2)}$ is a quasi-isometry invariant, and on cones and horns it equals one by [14], Lemma 3.4. Since $\chi_{(2)}$ is calculated on the smooth part $V_{0}$, one has $\chi_{(2)}\left(U_{\varepsilon^{\prime}}\right)=\chi_{(2)}\left(U_{\varepsilon^{\prime}} \backslash p_{1}, \ldots, p_{R}\right)=N$.

\section{REFERENCES}

[1] M. Atiyah, Resolution of singularities and division of distributions, Comm. Pure Appl. Math. 23 (1970), 145-150. MR 41:815

[2] I. N. Bernstein and S. I. Gel'fand, Meromorphic property of the functions $P^{\lambda}$ (English. Russian original) Funct. Anal. Appl. 3(1969), 68-69; translation from Funkts. Anal. Prilozh. 3 (1969), 84-85. MR 40:723

[3] E. Bierstone and P. D. Milman, Canonical desingularization in characteristic zero by blowing up the maximum strata of a local invariant, Invent. Math. 128 (1997), 207-302. MR 98e: 14010

[4] E. Bierstone and P. D. Milman, Semianalytic and subanalytic sets, Inst. Hautes Etudes Sci. Publ. Math. 67 (1988), 5-42. MR 89k:32011

[5] L. Birbrair, Local bi-Lipschitz classification of 2-dimensional semialgebraic sets, Houston J. Math. 25 (1999), 453-472. MR 2000j:14091

[6] L. Bröcker, M. Kuppe, and W. Scheufler, Inner metric properties of 2-dimensional semialgebraic sets, in: Real algebraic and analytic geometry (Segovia 1995), Rev. Mat. Univ. Complut. Madrid 10 (1997), 51-78. MR 98m:53084

[7] J. Brüning, Irregular spectral asymptotics, Canad. Math. Soc. Conf. Proc. 29 (2000), 85-99. MR 2002b:58032

[8] J. Brüning, The signature theorem for manifolds with metric horns, Journées: "Équations aux Dérivées Partielles" (Saint-Jean-de-Monts, 1996), Exposé II, École Polytech., Palaiseau, 1996. MR 98a:58150

[9] J. Brüning and M. Lesch, Hilbert complexes, J. Funct. Anal. 108 (1992), 88-132. MR 93k:58208

[10] J. Brüning and M. Lesch, Kähler-Hodge theory for conformal complex cones, Geom. Funct. Anal. 3 (1993), 439-473. MR 94i:58189

[11] J. Brüning and M. Lesch, On the spectral geometry of algebraic curves, J. reine angew. Math. 474 (1996), 25-66. MR 97d:58193

[12] J. Brüning, N. Peyerimhoff, and H. Schröder, The $\bar{\partial}$-operator on algebraic curves, Commun. Math. Phys. 129 (1990), 525-534. MR 91g:58273

[13] C. Callias, On the existence of small-time heat expansions for operators with irregular singularities in the coefficients, Math. Res. Letters 2 (1995), 129-146. MR 96h:58165

[14] J. Cheeger, On the Hodge theory of Riemannian pseudomanifolds, Proc. Sympos. Pure Math. 36 (1980), 91-146. MR 83a:58081

[15] J. Cheeger, Spectral geometry of singular Riemannian spaces, J. Diff. Geom. 18 (1983), 575657. MR 85d:58083

[16] J. Cheeger, M. Goresky, and R. MacPherson, $L^{2}$-cohomology and intersection homology of algebraic varieties, Annals of Math. Studies 102 (1982), 303-340. MR 84f:58005

[17] G. Eulering, Integrale Krümmungskonstanten algebraischer Untermannigfaltigkeiten von Räumen konstanter Krümmung, Doctoral Thesis, Münster 1995.

[18] J. Fu, Curvature measures of subanalytic sets, Amer. J. Math. 116 (1994), 819-880. MR 95g:32016

[19] I. M. Gel'fand and G. E. Shilov, Generalized Functions, Vol. I, Academic Press, New York, 1964. MR 29:3869]

[20] D. Grieser and M. Lesch, On the $L^{2}$ Stokes theorem and Hodge theory for singular algebraic varieties, to appear in Math. Nachr.

[21] D. Grieser, Quasiisometry of singular metrics, to appear in Houston J. Math.

[22] H. Hironaka, Resolution of singularities of an algebraic variety over a field of characteristic zero, Ann. of Math. 79 (1964), 109-326. MR 33:7333

[23] M. W. Hirsch, Differential Topology, Springer, 1976. MR 56:6669 
[24] P. Jeanquartier, Transformation de Mellin et développements asymptotiques, L'Enseignement Mathématique 25 (1979), 285-308. MR 81g:46049

[25] M. Lesch and N. Peyerimhoff, An index theorem for manifolds with metric horns, Comm. Partial Differential Equations 23 (1998), 649-684. MR 99d:58166

[26] J.-M. Lion and J.-P. Rolin, Intégration des fonctions sous-analytiques et volumes des sousensembles sous-analytiques, Ann. Inst. Fourier 48 (1998), 755-767. MR 2000i:32011

[27] M. Nagase, Hodge theory of singular algebraic curves, Proc. Amer. Math. Soc. 108 (1990), 1095-1101. MR 90h:14031

[28] R. M. Ruiz, The basic theory of power series, Vieweg 1993. MR 94i:13012

[29] M. Spivak, A comprehensive introduction to differential geometry, Vol. III-IV, Publish or Perish, 1975. MR 51:8962] MR 52:15254a

Institut für Mathematik, Humboldt Universität Zu Berlin, Sitz: Rudower Chaussee 25, 10099 Berlin, Germany

E-mail address: grieser@mathematik.hu-berlin.de

Current address: Department of Mathematics, Massachusetts Institute of Technology, 77 Massachusetts Ave., Cambridge, MA 02139 